\title{
ABSOLUTE PROPER MOTION OF GALACTIC GLOBULAR CLUSTERS
}

\author{
R.-D. SC'HOLZ \\ WIP Projehtgruppe Astrometrie \\ bei der Iniversität Potsdam. \\ An der Sternwarte, 16 \\ O-1590 Potsdam \\ Germany
}

\author{
M.J. IRWIN \\ Royal Greenwich Observatory \\ Madingley Road \\ Cambridge CB:3 OEZ \\ Great Britain
}

\begin{abstract}
ABSTRAC:T. Using automated scans of Tautenburg Schmidt plates with the APM facility in Cambridge (UK) the mean tangential motion of two globular clusters, M3 and M92, is determined directly with respect to a well defined extragalactic reference frame. In both fields five pairs of plates centred on the cluster with epoch differences from 20 to 27 years were measured. In the independent proper motion determination with 1200 to 2300 reference galaxies for each pair of plates we used 3rd order polynomials and a stepwise regression method. The mean absolute cluster p.m. corrected for systematic effects dependent on the coordinates was obtained with an accuracy of \pm 0.03 arcsec/century for M3 and \pm 0.06 arcsec/century for M92.
\end{abstract}

\section{Introduction}

Our present knowledge of the kinematics of the galactic globular cluster system is mainly based on radial velocity studies. The key to further progress lies in the determination of absolute proper motions. Webbink (1988) reviewed the efforts in both directions and encouraged new attempts to obtain absolute proper motions of globular clusters directly with respect to background galaxies and quasars.

Proper motions of numerous globular clusters have been investigated by (udworth and coworkers by measuring the relative motion of cluster stars with respect to field stars. This required various assumptions concerning the direction of the solar apex and the secular parallaxes of the chosen field stars around each cluster. Not only were the results affected by the choice of solar apex and motion but they were also afflicted by large errors due to the small number of field stars used $(\sim 50)$ and their inherent proper motion dispersion (eg. (udworth 1979). Although a very high accuracy ( $\pm 0.02 \mathrm{arcsec} / \mathrm{century}$ ) in the relative proper motion of the cluster stars was achieved, the uncertainty in the absolute proper motion of the whole cluster remained of the order of 0.1 to 0.4 arcsec/century. Brosche et al. (1983, 1985) used Lick stars with known absolute proper motion as reference stars to connect globular cluster motions to an extragalactic reference frame, whilst Tucholke (1989) derived the proper motion of globular clusters relative to the background of the 
SMC:

This work describes the first measurement of the absolute proper motion of two globular clusters directly with respect to a large number $(\sim 2000)$ of background galaxies. The Tautenburg Schmidt telescope has been taking photographic plates of globular clusters since the early 1960 's. With a 25 year baseline it is now possible to use this material to study the proper motions of these globular clusters. In each of the cluster fields with M:3 and M92 five pairs of Tautenburg Schmidt plates were processed on the APM facility in Cambridge. This system has already been extensively used for astrometric work using UK Schmidt and Palomar Schmidt photographic plates (Kibblewhite et al. 1982; Kibblewhite et al. 1984; Evans 1988).

\section{Observations and Measurements}

The Tautenburg Schmidt telescope with a $2 \mathrm{~m}$ mirror, a $1.34 \mathrm{~m}$ correction plate and a focal length of $4 \mathrm{~m}$ has a plate scale of $51.4 \mathrm{arcsec} / \mathrm{mm}$. With a useful plate size of $24 \mathrm{~cm}$, each plate covers some $3^{\circ} \times 3^{\circ}$ of sky, providing sufficient background galaxies to define an absolute inertial reference frame. The plate pairs selected for this project had epoch differences between 20 and 27 years (see Table 1 ). All plates were measured on the APM. The measured objects were classified into stars, nonstellar objects, noise images and merged objects using the standard APM software. An internal magnitude calibration as described by Bunclark and Irwin (1983) was also carried out. In a previous study (Scholz and Schmidt 1992) the APM magnitudes linearly related to photoelectric magnitudes were converted into B magnitudes using the photographic photometry of Bronkalla (1972) and photoelectric standards of Sandage and Walker (1965) and of Sandage and Katem (198:3).

For the plate matching of all 10 plates per field we used the deepest first epoch plate as the reference plate. The plate matching consists in pairing up objects between the reference plate and the comparison plates using several passes of ever decreasing search radius, starting with bright objects and large search radii (up to several $\mathrm{mm}$ ), finally iterating down to faint objects within a target search radius of $30 \mu \mathrm{m}$. With a 20 years base line this corresponds to a proper motion of $7.5 \mathrm{arcsec} /$ century, so that only very high proper motion objects were excluded.

In order to exclude objects that were unreliably recorded or measured (due to say excessive image crowding, dirt or emulsion defects) only those images classified as stars on all plates or as galaxies on at least the two deepest plates were used in subsequent analysis. The faintest stars and galaxies $(B>20)$ were not used both because of their rapidly increasing positional errors and the uncertainty in the image classification of galaxies neas the plate limit. Bright galaxies ( $\mathrm{B}<1 i$ ) were not used because of the possible misclassification of bright stars and double stars announced by Schilbach and Scholz (1992). Finally we decided not to use the bright cluster stars $(B<1 i)$. since even allowing for the extra information present in the membership probabilities of these stars obtained by Cudworth (1976 and 1979) possible magnitude dependent systematic errors could begin to dominate the mean cluster motion (cf. Schilbach and Scholz 1992). 
Table 1. Quality of the different plate pairs

\begin{tabular}{|c|c|c|c|c|c|c|c|}
\hline $\begin{array}{l}\text { Plate } \\
\text { pair }\end{array}$ & $\begin{array}{l}\Delta \mathrm{E}_{\mathrm{p}} \\
\text { [years] }\end{array}$ & $\begin{array}{l}\text { No. of } \\
\text { galax. }\end{array}$ & $\begin{array}{c}\mathrm{x} \text { solution } \\
\text { error } \\
{[\mu \mathrm{m}]}\end{array}$ & $\begin{array}{c}\mathrm{y} \text { solution } \\
\text { error } \\
{[\mu \mathrm{m}]}\end{array}$ & $\begin{array}{c}\text { error } \\
\text { x p.m. } \\
{[\operatorname{arcsec} / c e n t]}\end{array}$ & $\begin{array}{c}\text { error } \\
\text { y p.m. } \\
{[\operatorname{arcsec} / \text { cent }]}\end{array}$ & Weight \\
\hline \multicolumn{8}{|l|}{ M3 } \\
\hline 1 & 23.0 & 2163 & 7.2 & 6.7 & 0.035 & 0.032 & 1.4 \\
\hline 2 & 23.0 & 1947 & 7.7 & 7.7 & 0.039 & 0.039 & 1.3 \\
\hline 3 & 19.9 & 1230 & 9.0 & 9.2 & 0.066 & 0.068 & 0.7 \\
\hline 4 & 20.2 & 1761 & 8.3 & 8.4 & 0.050 & 0.051 & 0.9 \\
\hline 5 & 20.2 & 1383 & 10.5 & 9.7 & 0.072 & 0.066 & $0 . \bar{\imath}$ \\
\hline \multicolumn{8}{|l|}{ M92 } \\
\hline 1 & 22.7 & 2346 & 5.9 & 6.2 & 0.028 & 0.029 & 1.0 \\
\hline 2 & 26.0 & 1539 & 6.5 & 6.8 & 0.033 & 0.034 & 1.0 \\
\hline 3 & 26.1 & 2236 & 7.3 & 7.6 & 0.030 & 0.032 & 1.0 \\
\hline 4 & 22.7 & 2203 & 7.2 & 7.4 & 0.035 & 0.036 & 1.0 \\
\hline 5 & $26 . \bar{i}$ & 1512 & 6.9 & 7.3 & 0.034 & 0.036 & 1.0 \\
\hline
\end{tabular}

\section{Reduction}

The global plate-to-plate transform models for each pair of plates were obtained with different samples of reference galaxies using 3rd order polynomials and the method of stepwise regression described in Hirte et al. (1990). Only coordinate dependent terms are included and only statistically significant terms are retained. Magnitude dependent terms were omitted because the magnitude interval for the objects used had already been restricted. The reference galaxies were defined by the requirements: that on the two deepest plates they be classified as galaxies, that they lay outside a radius of 8 arcmin (M92) or of 16 arcmin (M3) from the cluster centre, and that they be in the interval from $B=17$ to one magnitude above the plate limit to ensure reliable classification.

The number of galaxies ( see Table 1) depends on the limiting magnitudes of the plates in a pair, and consequently on the different quality of the images which influences the matching with the reference plate. The overall limiting magnitude was $B=21.3$ in the M3 field and B $=20.5$ in the M92 field. An estimate of the quality of the plate pairs can be obtained directly by dividing the unit weight error of the plate-to-plate solution by the epoch difference and the square root of the number of galaxies used in the solution. This formal error in defining an inertial reference frame in the field centre where the cluster is situated was used to compute weights for the plate pairs (Table 1). Whereas different weights had to be given to the M3 plate pairs mainly due to the lower plate limit of one first and one second epoch plate, there was no need to do so in the case of M92, because the plate-to-plate solutions were more accurate and lower numbers of reference galaxies were compensated by higher epoch differences.

Kibblewhite et al. (1982) and Evans (1988) described another astrometric reduction method for the APM measurements, whereby any systematic errors on scales of $1-2 \mathrm{~cm}$ are detected and then removed after first applying a linear plate-to-plate solution. Likewise any systematic errors caused by column scanning the plates in strips $2 \mathrm{~mm}$ wide are 
also estimated and removed. The general idea being to locally model any systematic error whatever the cause, eg. measuring machine, differential field distortion, plate emulsion non-elasticity etc., and simply subtract them off. Although this approach works well for studies of galactic structure (the original motivation) where the mean proper motion of stars over this size of field is essentially independent of position, for analysing the proper motion of a globular cluster, which itself only covers a few $\mathrm{cm}$ of plate, a modification of this method is required. However, since all the plates were taken using the same telescope setup and were of relatively short exposure times, high order plate-to-plate corrections (or rubber membrane type corrections) were found not to be needed (except for one of the M92 plates).

Consequently, we looked for systematic effects in the $x$ and $y$ proper motions dependent on the $x$ and $y$ position obtained from the global polynomial method. Periodic errors like the so called "2cm wobble" (Evans 1988. Schilbach and Scholz 1992) may not change the mean proper motion of the stars uniformly distributed over the whole field but they have to be considered in the case of a globular cluster with rather different number densities in dependence on the coordinates. So we assumed a constant proper motion of the field stars outside a given cluster radius and another constant proper motion for the cluster stars inside this radius with equal systematic errors of the $x$ and $y$ proper motions in dependence on the $x$ and $y$ position for both the cluster and the field stars. Binning the cluster and field stars' proper motions along the $x$ and $y$ axes, the corrected mean cluster proper motion was obtained summing up the mean field star proper motion and the mean difference of corresponding bins.

\section{Results}

The main results are listed in Table 2 . In the case of M92 we decided not to use the third pair of plates ( the second epoch plate had already been problematic in the plate matching with high order corrections needed) showing larger periodic effects obviously due to the measurements than all other pairs of plates. For both clusters the corrected $x$ and $y$ values of the absolute proper motions from all pairs of plates were in a better agreement than the uncorrected ones. The correction improved the solution even if the third M92 pair was included but a residual systematic shift in the $y$ proper motion probably introduced by the high order correction could not be removed.

The results compared with those of ('udworth (1976 and 1979) are shown in Figure 1. In the case of M92 our results are in good agreement with the absolutisation of Cudworth $(1976)$ when he used the standard apex. Also shown in the Figure are the directions of the standard antapex, the old Lick antapex (Klemola and Vasilevskis 1971), the new Lick antapex (Hanson 1987) and the antapex for the (ialactic globular cluster system taken from Mihalas and Binney (1981). There is a significant difference between the field and cluster proper motions. As expected there is a general trend of increasing proper motions of the field stars for brighter samples in the M3 field. In the M92 field which is closer to the apex this effect is not present.

Both absolute cluster proper motions obtained lie along the direction of the new Lick antapex. Adopting a radial velocity of $-150 \pm 5 \mathrm{~km} / \mathrm{s}$ and of $-118 \pm 5 \mathrm{~km} / \mathrm{s}$, and a distance 
Table 2. Mean absolute proper motions of M3 and M92

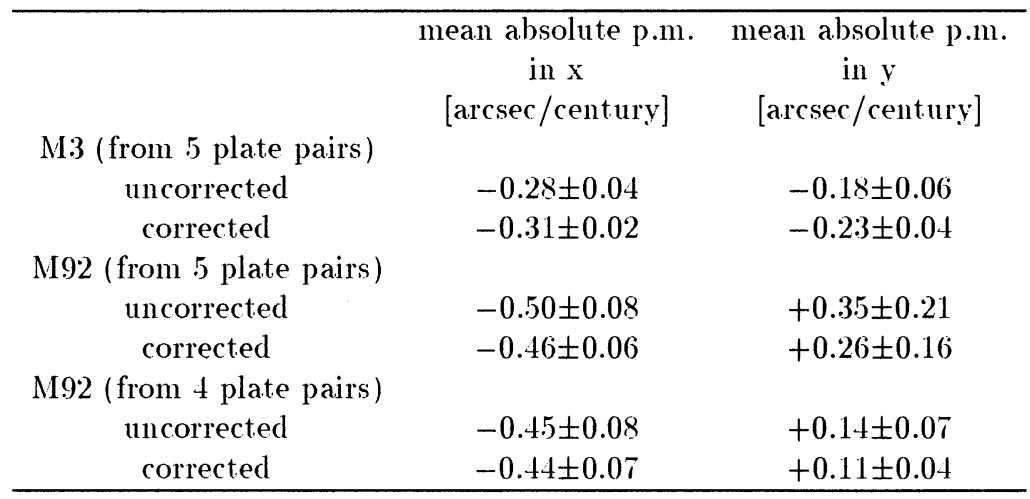
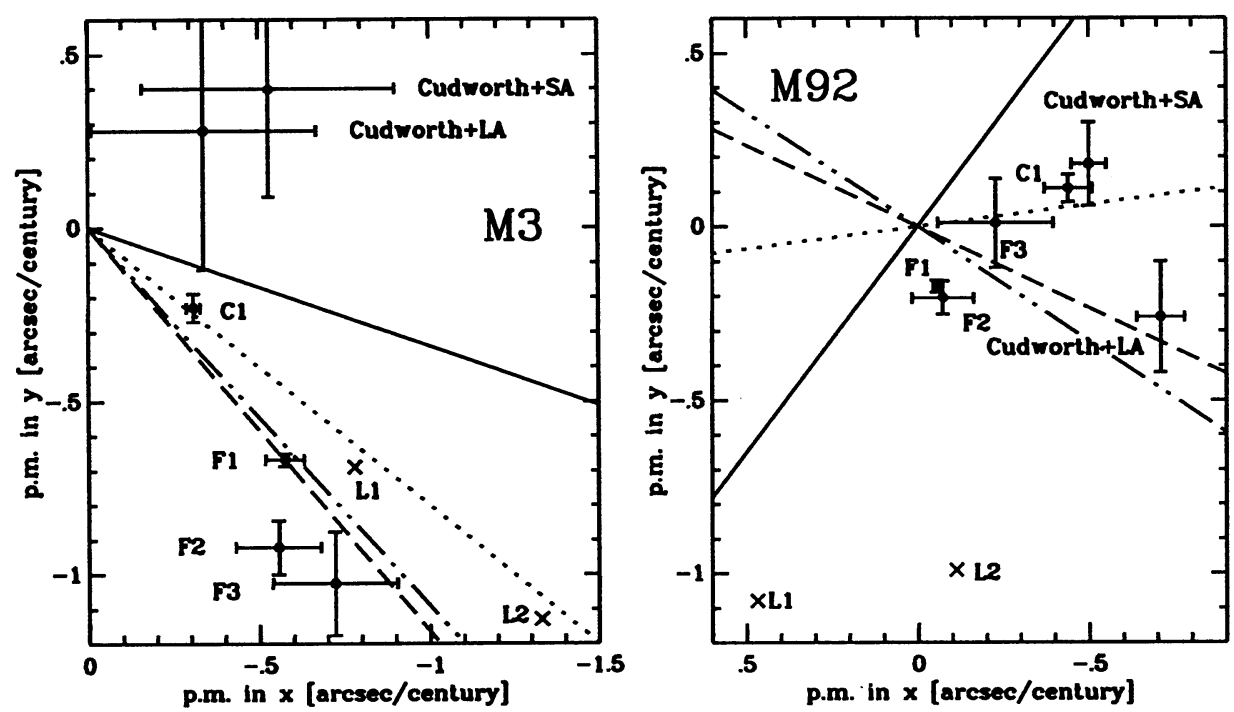

Figure 1. Mean absolute p.m. of cluster stars and field stars

Points corresponding to field and cluster stars together with their $1 \sigma$ error bars are marked. C.1 is the corrected cluster motion for $1032 \mathrm{M} 33$ stars and $416 \mathrm{~N}$.92 stars with $1 \mathrm{~T}<B<20$; F1 is the mean p.m. of 2990 and 6466 field stars with corresponding magnitudes; F2 is the mean p.m. of 1121 and 3823 field stars with $14<B<1 \bar{\tau}$; F3 is the mean p.m. of 220 and 580 field stars with $B<14$; respectively in the MI3 and $\mathrm{M} 92$ fields. The points labelled L1 and L2 are Lick proper motion measurements for about 50 faint and bright stars respectively taken from Klemola and Vasilevskis ( 1971$)$. The lines represent: solid - standard antapex; dashed - solar antapex with respect to globular clusters; dot-dash - old Lick solar antapex; dotted - new Lick solar antapex. 
of $11.0 \pm 1.5 \mathrm{kpc}$ and of $9.0 \pm 1.5 \mathrm{kpc}$, we obtain heliocentric galactic space velocities according to Johnson and Soderblom $(1987)$ of $(\mathrm{U}, \mathrm{V}, \mathrm{W})=(-70,-212,-114) \pm 18 \mathrm{~km} / \mathrm{s}$ and $(-100,-183,+90) \pm 23 \mathrm{~km} / \mathrm{s}$, respectively for M3 and M92. A more detailed discussion of the space motion in dependence on the solar motion will be given later.

In future work we intend to extend the determination of absolute proper motions to other globular clusters and to some of the Galactic dwarf spheroidal satellites to fully exploit the available Tautenburg plate material.

\section{ACKNOWLEDGEMENTS}

We would like to thank the Karl-Schwarzschild-Observatorium Tautenburg for supplying the plates. This work has been supported by the Koordinierungs- und Aufbau-Initiative für die Forschung. R. Scholz would like to thank the Institute of Astronomy, Cambridge for hospitality and financial support during some of this work.

\section{REFERENCES}

Bronkalla, W. : 1972, Thesis, Potsdam.

Brosche, P., Geffert, M., Ninkovic, S.: 1983, Publ. Astron. Inst. Czech. Acad. Sci, 56. 145. Brosche, P., Geffert, M., Klemola, A.R., Ninkovic, S.: 1985, Astron.J. 90. 2033.

Bunclark, P.S., Irwin, M.J.: 1983, In: E.J. Rolfe (ed.): Statistical Methods in Astronomy. Proc. of an internat. Coll., Strasbourg 13-16 Sept.1983, (ESA SP-201), p.195.

Cudworth. K.M.: 1976, Astron.J. 81, 975.

Cudworth, K.M.: 1979, Astron.J. 84, 1312.

Evans, D.W.: 1988, Dissertation, Cambridge, 189 p.

Hanson, R.B.: 1987, Astron.J. 94, 409.

Hirte, S., Dick, W.R., Schilbach, E., Scholz, R.-D.: 1990, In: C. Jaschek and F. Murtagh (eds.): Errors, Uncertainties and Bias in Astronomy, Cambridge University Press. p. 343.

Johnson, D.R.H., Soderblom, D.R.: 1987, Astron.J. 93, 864.

Kibblewhite, E.J., Irwin, M.J., Bridgeland, M.T., Bunclark, P.S.: 1982, Occ. Rep. R. Obs. Edinburgh, 10, 79.

Kibblewhite, E., Bridgeland, M., Bunclark, P., Cawson, M., Irwin, M.: 1984, in: M. (apaccioli (ed.): Astronomy with Schmidt-Type Telescopes. Reidel Publ.. p. 89.

Klemola, A.R.. Vasilevskis, S.: 1971. Publ. Lick Obs.. XXII. part III.

Mihalas, D., Binney, J.: 1981. Calactic Astronomy. Freeman. San Francisco.

Sandage, A., and Katem, B. : 1983, Astron.J. 88. 1146.

Sandage, A., and Walker, M.F. : 1966, Astrophỵs.J. 143, 313.

Schilbach, E.. Scholz, R.-D.: 1992, Astron. Nachr. 313, 243.

Scholz, R.-D., Schmidt, K.-H.: 1992. Astron. Nachr. 313, 45.

Tucholke, H.J., 1989, PhD Thesis, Münster.

Webbink, R.F, 1988, In: J.E. Grindley, A.G. DavisPhilip (eds.): The Harlow Shapley Symposium on Globular Cluster Systems in Calaxies - IAU Symp. 126, p 49. 